\section{Les papillomavirus humains cutanés oncogènes sont-ils des commensaux défectifs?}

Maciej Lazarczyk, Michel Favre
M. Lazarczyk : Unité de Génétique,

Papillomavirus et Cancer Humain,

Institut Pasteur, 75015 Paris, France.

Inserm U563, CPTP et Université Paul Sabatier,

31300 Toulouse, France.

M. Favre : Unité de Génétique,

Papillomavirus et Cancer Humain,

Institut Pasteur, 25, rue du Docteur Roux,

75724 Paris Cedex 15, France.

mfavre@pasteur.fr

téine $\mathrm{TMCl}$, on peut émettre l'hypothèse que les protéines $\varepsilon V \varepsilon R$ jouent un rôle dans le transport d'oligoéléments (potassium, calcium, zinc...) ou dans la transmission de signaux cellulaires.

\section{Protéines EVER et homéostasie du zinc} Pour tenter d'élucider la fonction des protéines $\varepsilon V \varepsilon R 1$ et $\varepsilon V \varepsilon R 2$, nous avons entrepris d'identifier leurs protéines cellulaires partenaires par une méthode de double hybride chez la levure [5]. Nous avons montré que les protéines EVER forment un complexe avec ZnT-1, une protéine transporteuse de zinc. Le zinc est un oligoélément extrêmement toxique sous forme libre dans la cellule. Aussi, certaines protéines cellulaires comme les métallothionéines 1 et 2 sont capables de fixer le zinc libre alors que d'autres, appartenant à la famille ZIP ou ZnT, interviennent dans l'import ou l'export cellulaire du zinc ou dans son immunocompétents ou immunodéprimés pourrait indiquer un rôle de ces virus dans la carcinogenèse cutanée [3].

II y a maintenant quelques années, nous avons identifié deux gènes ( $E V \varepsilon R I$ et EVER2) dont des mutations homozygotes confèrent la sensibilité à l'infection virale des patients atteints d'દV [4]. Ces deux gènes, encore appelés respectivement TMC6 et TMC8, appartiennent à la famille des protéines TMC (transmembrane channel-like proteins). Les protéines $\varepsilon V \varepsilon R 1$ et EVER2 comportent respectivement 10 et 8 domaines transmembraires putatifs et sont localisées au niveau du réticulum endoplasmique (Figure 1). Leur fonction est inconnue, mais comme pour la pro-

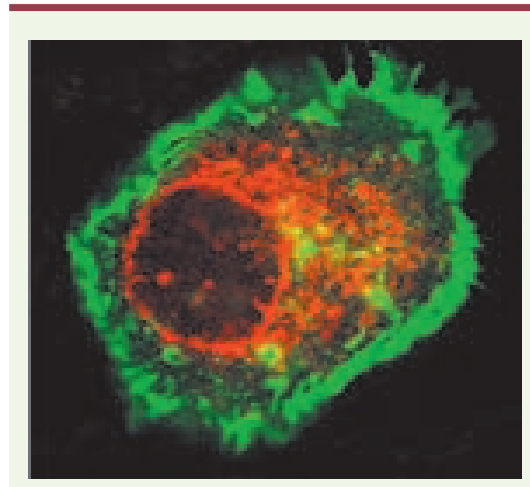

Figure 1. La protéine EVER2 (en rouge) est localisée dans la membrane nucléaire et dans le cytoplasme des kératinocytes. La membrane plasmique est colorée en vert. 
transport intracellulaire [6]. Il a également été montré que le zinc était capable d'induire la synthèse des métallothionéines et de ZnT-1, permettant ainsi de réduire la quantité de zinc libre. Dans les kératinocytes, la cellule hôte des $\mathrm{PVH}$, le complexe $\varepsilon V \varepsilon R / Z n T l$ n'est pas mis en jeu dans l'efflux du zinc. En revanche, nous avons montré que la protéine દVદR2 régule la distribution du zinc libre et inhibe son transport dans le noyau vers le nucléole qui est le siège d'une transcription active des ARN ribosomaux [5]. De fait, les kératinocytes possédant un gène $E V \varepsilon R 2$ muté ont une croissance plus rapide que les cellules normales. Nous avons également montré que le complexe EVER/ZnTl réprime l'activité du facteur de transcription MTF-1 (metal-responsive transcription factor-1), dont la synthèse dépend du zinc et qui active la transcription des métallothionéines. De même, les protéines EVER et $\mathrm{ZnT}-1$ inhibent les voies de signalisation des MAP kinases (mitogen-activated protein kinases) qui conduisent à la synthèse de facteurs de transcription comme c-Jun et c-Fos de la famille AP-1. Ces facteurs jouent un rôle très important dans la synthèse de cytokines et chimiokines et dans la transcription des génomes de PVH.

Aussi, au cours de l'infection virale, la levée de cette inhibition exercée par le complexe EVER/ZnT-1 pourrait jouer un rôle important dans la réponse immune des kératinocytes contre les infections par des PVH $\beta$, ou dans le contrôle intracellulaire de l'expression du génome viral ou de l'activité des protéines virales précoces comme les protéines $\varepsilon 6$ et $\varepsilon 7$.

\section{Protéines EVER \\ et contrôle de l'infection virale}

Nous étudions actuellement l'impact de mutations dans les protéines EVERl ou EVER2 dans le profil d'expression de cytokines et de chimiokines de lignées lymphoblastoïdes et de kératinocytes isolés de patients et de sujets sains de familles atteintes d'eV.

Dans le but de démontrer un contrôle intracellulaire de l'expression ou de l'activité des protéines virales précoces, nous avons tenté de mettre en évidence une interaction entre les protéines $\varepsilon V \varepsilon R$ ou ZnT-1 avec des protéines virales précoces des PVH cutanés et génitaux. Cette étude était d'autant plus justifiée qu'une



Figure 2. Modèle proposé pour la restriction de l'expression des PVH $\beta$ dans les kératinocytes de sujets normaux ou atteints d'épidermodysplasie verruciforme. A. Dans un kératinocyte normal, l'activation par le zinc de la synthèse de facteurs de transcription de la famille AP-1 est contrecarrée par le complexe EVER1/EVER2/ZnT-1. L'infection de la cellule par le PVH16 permet la synthèse de la protéine 16 \&5 qui vient lever l'inhibition du complexe EVER/ZnT-l et favoriser l'expression des facteurs de transcription nécessaires à la transcription et la réplication du génome viral. B. L'absence d'un gène $\varepsilon 5$ dans le génome des PVH $\beta$ ne permet pas de lever cette inhibition, ce qui pourrait expliquer l'absence de réplication végétative de ces virus et de lésions dans la population générale. C. En revanche, dans l'épidermodysplasie verruciforme, une mutation dans le gène $E V E R I$ ou dans le gène $\varepsilon V E R 2$ bloquerait la formation du complexe EVER/ZnT-1 et permettrait l'expression des facteurs de transcription de la famille AP-l et la réplication virale. 
Les PVH ont la particularité de se répliquer dans les kératinocytes en voie de différenciation terminale. Dans les lésions associées au PVH16, 16 \&5 est essentiellement décelée dans les couches basales et parabasales de l'épiderme. Cela suggère que cette protéine joue un rôle prépondérant, en conjonction avec les protéines $\varepsilon 6$ et $\varepsilon 7$, dans la prolifération des cellules basales et dans la réinitiation, au cours de l'étape de différenciation, de la phase $S$ du cycle cellulaire pendant laquelle sont synthétisées les ARN et ADN polymérases nécessaires à la réplication virale. II a également été rapporté que 16 \&5 est capable, par la voie PKC et les voies de signalisation dépendantes de ras, de stimuler la synthèse de facteurs de transcription c-Jun, Jun B et c-fos [8]. Étant donné que plusieurs sites de fixation de ces facteurs sont localisés dans la longue région de régulation des $\mathrm{PVH}$, on peut penser que 16 $\varepsilon 5$ joue un rôle essentiel dans l'activation de la transcription des gènes viraux, en particulier les gènes de transformation $\varepsilon 6$ et $\varepsilon 7$ et les gènes de réplication $\varepsilon l$ et $\varepsilon 2$.

\section{Les PVH $\boldsymbol{\beta}$, des commensaux défectifs}

II avait été préalablement montré que la protéine 88 du papillomavirus du lapin cottontail, une protéine possédant une structure et des propriétés analogues à celles de la protéine $\varepsilon 5$, était également capable d'interagir avec ZnT-l et, de ce fait, jouer un rôle important dans l'ho- méostasie du zinc et dans le cycle de réplication du virus [7]. Ainsi, selon le type de papillomavirus, deux protéines virales (દ5 et $\varepsilon 8$ ) pourraient interférer avec la régulation du zinc et favoriser l'activation de voies de signalisation impliquées dans la synthèse de facteurs de transcription essentiels pour l'expression et la réplication du génome viral (Figure 2). Parmi les PVH cutanés ou muqueux, les PVH du genre $\beta$ se distinguent par l'absence des gènes $\varepsilon 5$ et $\varepsilon 8$ [9]. Ainsi, nos résultats viennent corroborer l'hypothèse récemment formulée [2] selon laquelle les PVH $\beta$, en particulier le PVH5 oncogène, sont des commensaux défectifs très répandus et asymptomatiques dans la population générale. Seules une mutation dans les gènes $\varepsilon V \varepsilon R 1$ ou $\varepsilon V \varepsilon R 2$ ou des conditions physiologiques particulières, comme la régénération de l'épiderme [10], sont capables de permettre leur réplication et de lever la restriction d'hôte observée dans la population générale. Ainsi, que ce soit par une protéine virale ou par une mutation dans une protéine cellulaire, l'homéostasie du zinc semble jouer un rôle primordial dans l'infection par les papillomavirus. II convient donc maintenant de déterminer la place à accorder aux propriétés antivirales des protéines $\varepsilon V \varepsilon R$ dans le traitement des lésions associées aux $\mathrm{PVH}$, en particulier les $\mathrm{PVH}$ oncogènes. $\diamond$ Are oncogenic cutaneous HPVs defective commensals?

\section{REMERCIEMENTS}

Contrats de la Ligue Nationale contre le Cancer ( $n^{\circ} R 05 / 75-129$ et $n^{\circ}$ RS07/75-75) et de l'Association de la Recherche contre le Cancer ( $n^{\circ} 3731$ XA0531F et $n^{\circ} 4867$ ). M.L. est financé par une bourse de l'ARC et par la Foundation for Polish Science

\section{RÉFÉRENCES}

1. Schiffman M, Castle PE, Jeronimo J, et al. Human papillomavirus and cervical cancer. Lancet 2007 ; $370: 890-907$.

2. Orth G. Genetics of epidermodysplasia verruciformis : insights into host defense against papillomaviruses. Semin Immunol 2006; 18: 362-74.

3. Forslund 0 , Iftner T, Andersson K, et al. Viraskin study group. Cutaneous human papillomaviruses found in sunexposed skin : beta-papillomavirus species 2 predominates in squamous cell carcinoma. J Infect Dis 2007 ; $196: 876-83$

4. Ramoz N, Rueda LA, Bouadjar B, et al. Mutations in two adjacent novel genes are associated with epidermodysplasia verruciformis. Nat Genet 2002 ; 32 : 579-81.

5. Lazarczyk M, Pons C, Mendoza JA, et al. Regulation of cellular zinc balance as a potential mechanism of EVERmediated protection against pathogenesis by cutaneous oncogenic papillomaviruses. J Exp Med 2008 ; 205 : 35-42.

6. Palmiter RD. Protection against zinc toxicity by metallothionein and zinc transporter 1. Proc Natl Acad Sci USA 2004 ; 101 : 4918-23.

7. Nonnenmacher M, Salmon J, Jacob Y, et al. Cottontail rabbit papillomavirus $\varepsilon 8$ protein is essential for wart formation and provides new insights into viral pathogenesis. J Virol $2006 ; 80: 4890-900$.

8. Tsai TC, Chen SL. The biochemical and biological functions of human papillomavirus type $16 \varepsilon 5$ protein. Arch Virol $2003 ; 148: 1445-53$

9. Bravo IG, Alonso A. Mucosal human papillomaviruses encode four different $\varepsilon 5$ proteins whose chemistry and phylogeny correlate with malignant or benign growth. J Virol $2004 ; 78: 13613-26$

10. Favre M, Majewski S, Noszczyk B, et al. Antibodies to human papillomavirus type 5 are generated in epidermal repair process. J Invest Dermatol 2000 ; 114 : 403-7.

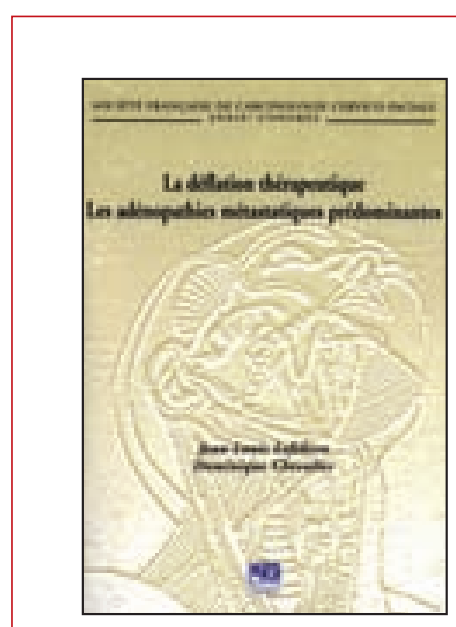

ISBN : 978-2-8425-4121-7 202 pages

\section{Bon de commande}

À retourner à EDK, 2, rue Troyon - 92316 Sèvres Cedex

Tél. : 0155641393 - Fax : 0155641394 - E-mail : edk@edk.fr

NOM : Prénom :

\section{Adresse :}

Code postal : Ville :

Pays :

Fonction :

Je souhaite recevoir l'ouvrage La déflation thérapeutique. Les adénopathies métastatiques prédominantes : $35 €+3 €$ de port $=\mathbf{3 8} € \mathbf{T T C}$

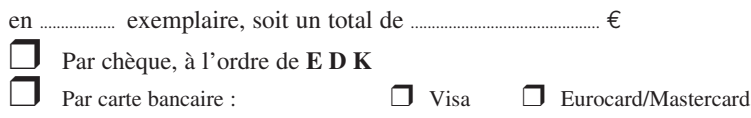

Date d'expiration: $\quad|\quad| \quad|\quad| \quad \mid$

$\mathrm{N}^{\circ}$ de contrôle au dos de la carte : । | | 